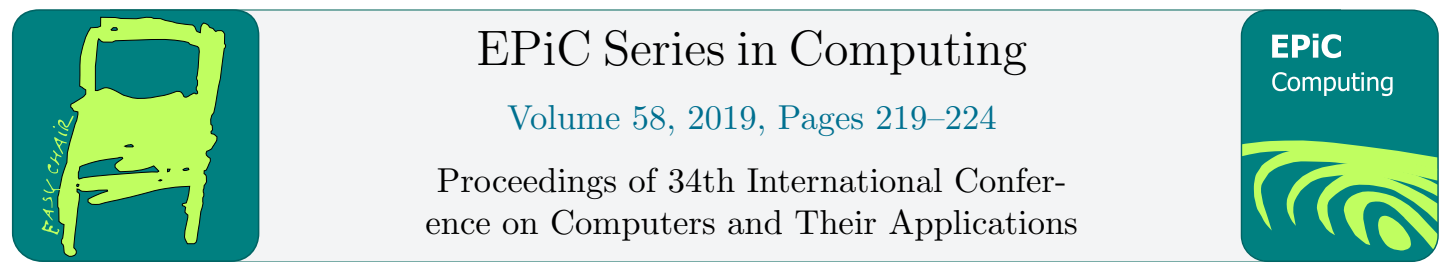

\title{
Smart Mobile Computing in Pregnancy Care *
}

\author{
Francisca Fonseca ${ }^{1}$, Hugo Peixoto ${ }^{2}$, Jorge Braga ${ }^{3}$, José Machado², and António \\ Abelha ${ }^{2}$ \\ 1 University of Minho, Campus Gualtar, Braga 4710, Portugal \\ a71934@alunos.uminho.pt \\ 2 Algoritmi Center, University of Minho, Campus Gualtar, Braga 4710, Portugal \\ \{hpeixoto, jmac, abelha\}@di.uminho.pt \\ 3 Centro Hospitalar do Porto, Centro Materno Infantil, Porto 4099, Portugal \\ jorgesousabraga@gmail.com
}

\begin{abstract}
Pregnancy is a period of changes. With all the information available and all the questions raised, it may also be an overwhelming period. Mobiles phones might be a solution for pregnant women to follow their pregnancy through Electronic Maternity Records (EMR). Therefore, this paper aims to propose an EMR to help women during their pregnancy. Firstly, the importance of Personal Health Records (PHRs) as well as mHealth is overviewed. Secondly, the types of mobile apps are presented with their pros and cons and the concept of Progressive Web App (PWA) is introduced. In order to understand the features that pregnancy mobile apps are now offering and the ones they are missing, eight apps are analysed. Lastly, the features and architecture of the proposed EMR are described and discussed. Since PWAs are a recent technology and a promising alternative to the three classic types of mobile development, it is also the technology used to develop the proposed EMR.
\end{abstract}

\section{Introduction}

The advancements in healthcare practice led to an increasing of the demand for electronic health systems also known as eHealth systems [9]. Personal Health Records (PHRs) aims to provide the patient with the ability to safely and confidentially access, manage and share their health information [11]. PHRs supply patients and healthcare providers with a complete summary of patient's health history and empower patients to have a more active role on their own health, to improve their health literacy with complete and accurate health information, to better communicate with their healthcare providers and to practice preventive self-care routines $[7,11]$. Health systems quality of diagnosis and quality of patients' treatment are also improved when PHRs are exchanged [17].

A sub-segment of eHealth systems is mHealth (mobile Health), where health interventions

*This work has been supported by FCT - Fundação para a Ciência e Tecnologia within the Project Scope: UID/CEC/00319/2019.

G. Lee and Y. Jin (eds.), CATA 2019 (EPiC Series in Computing, vol. 58), pp. 219-224 
through mobile devices used in medicine and public health are implemented [3, 19]. mHealth was defined by the Global Observatory for eHealth, and referred in the 2011 World Health Organisation report, as "medical and public health practice supported by mobile devices, such as mobile phones, patient monitoring devices, personal digital assistants (PDAs), and other wireless devices" [12]. Some studies show the success of mHealth interventions. In New Zealand, for example, a study has shown how receiving simple text message interventions would make the smokers twice more likely to quit smoking on the following six months [21]. Moreover, Widmer (2015) has also shown how using a mobile app by patients with coronary disease who were on rehabilitation would improve significantly their health and decrease the rehospitalisation and emergency department visits [16].

Electronic Maternity Records (EMRs) are a specific type of PHRs used for maternal care. EMRs are usually a way of exchanging pregnant women's health data between healthcare providers $[4$, 13]. The expansion of smartphones and mobile apps has been enormous and to take advantage of those tools to develop a mobile app for pregnant women, which has an EMR integrated, might be an efficient and easy way to provide antenatal care and to monitor pregnancy.

Through this paper, some pregnancy apps that are available on stores at the present day are analyzed. Besides, using the Progressive Web App technology and taking advantage of the user's experience that it provides, it will be suggested a methodology to develop an alternative personal antenatal care information system where women can access to their EMR and monitor their pregnancy.

\section{State of the Art}

\subsection{Mobile Development}

The technology used for mobile development as well as the operating system where the mobile apps are capable of running on define three types of mobile apps: native app, web app and hybrid app. The first one, native apps, is created with the tools and programming language characteristic of a certain platform, thereby they are developed for a specific operating system [8]. Besides being available on stores, native apps not only have a higher performance but also the look and feel of the operating system, creating a great user interface [8, 14]. However, it takes a higher level of experience to be developed directly upon the mobile platform services and the platform-specific API [18].

Mobile web apps are implemented by using easy to learn and develop web technologies, like HTML 5, JavaScript and CSS, which are hosted on remote servers and served via standard protocols $[8,14]$. A web app is a mobile-optimized website that will use the web mobile browser to run offering a uniform experience across platforms [14]. However, web apps are not available at any store, they are accessed via the URL and need internet connection to run the app [8].

The third and last type of mobile apps, hybrid apps, is a mixture of the best characteristics of native and hybrid apps. They are implemented with the same standard web technologies used to develop mobile web apps and also with a hybrid development framework [8, 14]. This framework makes it possible to create a cross-platform web-based mobile app, because developers are given access to a web-based code wrapper and also to a generic JavaScript API as a cross over between the web-based code and the corresponding platform API. The amount of time, cost and effort to develop and maintain a hybrid app is lower than the time, cost and effort needed to develop and main the previous presented native apps [8].

In 2015 a new type of web app, the Progressive Web App (PWA), which aimed to heighten the mobile web experience by seizing new features supported by the modern browsers, was 
introduced. The first time one accesses to a PWA it is through the browser like a classic web app. Only then the user can decided to install the app and an icon will be added to the home screen. Like native and hybrid apps and unlike web apps, push notifications, running in a full-screen mode and working offline are also possible when the PWA is installed. Yet, the development of a PWA, since it is made with web technologies, is easier and quicker than developing a native and a hybrid app [14].

\subsection{Electronic Maternity Records and Pregnancy Mobile Apps}

There is a great need of information during the 9-month period of pregnancy. Studies have shown that a large percentage of pregnant women around the world use the Internet as a source of information on pregnancy, complementary to the information from professionals, where foetal development, nutrition and childbirth are the most common subjects [15]. Other searched topics are delivery complications, pain management and physical exercise during pregnancy. Moreover, providing future mothers the access to some information on their pregnancy might calm, inform and make them more comfortable about it $[4,10]$.

In 2016, Bachiri et al. in [3] analysed 33 pregnancy mobile apps. At that time, they concluded that to have an accurate and complete pregnancy tracker, the pregnancy mobile apps should contain the greatest amount of pregnancy areas. They have also reached the conclusion that the features of the mobile pregnancy apps must be adapted to their users' needs. Therefore, the current version available on stores of the apps classified by Bachiri et al. in their 2016 study in [3] with the highest score (above 15 points) are analysed in this paper. These apps are:

- "Glow Nurture - Pregnancy App" (Android and iOS);

- "Gestavida Pregnancy" (Android and iOS);

- "WebMD Pregnancy" (iOS);

• "Ovia Pregnancy Tracker \& Baby Countdown Calendar" (Android and iOS);

- "Pregnancy ++" (Android and iOS);

- "BabyCentre - My Pregnancy \& Baby Today" (Android and iOS);

- "WomenLog Pregnancy" (Android);

- "iPregnant Prengnacy Tracker Free" (iOS).

Overall, there are two common features to all the 8 previous pregnancy apps. They all provide a birth date calculator and weekly information on foetal development and/or mother's body. "WomanLog Prengnacy" is mainly a monitoring app, the information on foetal development is few and it is the only app that doesn't give any suggestion and tips about healthy habits. However, "WomanLog Pregnancy" has a calendar where each day the user may record time and duration of the contractions, number of foetal movements, medical appointments, moods, weight, waistline and symptoms. From measurements such as weight and blood pressure to lifestyle like physical activity and meals, each one of the previous apps supplies at least one monitoring or tracking tool. If the user has diabetes or is diagnosed with gestational diabetes, only "Gestavida Pregnancy" supplies a tool to monitor glucose levels. Apps such as "WebMD Pregnancy", "BabyCentre - My Pregnancy \& Baby Today" and "iPregnant Pregnancy Tracker Free" also provide a community forum and "Pregnancy ++ " allows the medical appointments 
record. Moreover, "Gestavida Pregnancy" and "Ovia Pregnancy Tracker \& Baby Countdown Calendar" are the only apps where the user can record her medical history. In spite of being one of the most complete apps, "Ovia Pregnancy Tracker \& Baby Countdown Calendar" has the main setback of not working offline. "WebMD Pregnancy", "WomanLog Pregnancy" and "iPregnant Pregnancy Tracker Free" are only available to one operating system limiting the number of users.

\section{The Proposed Electronic Maternity Record Application}

The personal antenatal care information system here proposed aims to support future mothers during their pregnancy by allowing them to record personal information and medical history or to have access to pregnancy-related information. However, women who are trying to conceive as well as mothers who just had their babies may find some answers in the developed system. The features provided by the proposed app can be grouped in: 1) Maternity records and appointments - the maternity record used in our system is based on the Portuguese pregnancy booklet "Boletim de Saúde da Grávida". 2) Health education - health and pregnancy-related based on their gestational age and health conditions recorded on the previous pregnancy booklet. There is also a section of frequently asked questions (FAQs) regarding the become pregnant, pregnancy and post-partum periods. 3) Pregnancy-related information - the information on foetal development as well as the changes occurring on pregnant women's body are updated every week. 4) Monitoring and tracking tools - users have access to weight, blood pressure, foetal movements and glucose monitoring tools as well as moods, meals, physical activity and milestones trackers.

The main purpose of any app is to be available to the widest range of users. Furthermore, the success of any mobile app depends on what it does, how it is done and how it is perceived by its users. In order to overcome some resistance of mHealth apps adoption, it is needed to ensure a good User Experience (UX). To accomplish all that and due to the great UX it grants, the methodology chosen was that of the PWAs.

The user's interface was built with the open-source library React. It is declarative and component-based and a great tool to avoid the cascade of updates that may happen with the growing of the applications complexity. Each component controls its own state and renders itself only when changed [2]. React doesn't manipulate directly the DOM when the data changes. Instead, the view looks is declared using the virtual DOM technology. Only the computed differences between the real and the virtual DOM will be applied to the real DOM. This results on a faster loading of the HTML pages with little overhead [20].

The frontend makes HTTP requests to the server on the backend. Node.js was the runtime environment chosen and Express.js was the web server framework adopted to write the server code. The server in Node.js analyses the request and, then, the Express.js works as a middleware and asks the database, which was the NoSQL document-oriented MongoDB, for the data. Express.js allows defining routes which will have an associated function to respond to the arrived HTTP users' request. The requests made by the frontend parse URL, headers, bodies and parameters. By using MongoDB the load is distributed over multiple servers and the database is able to scale with the terabytes of users' data that may need to be stored in web-oriented systems [5]. At the database of the proposed app, pregnancy-related and health information as well as FAQs and users' data are saved.

The service worker (SW), the JavaScript file that runs in parallel with the main browser thread and is characteristic of the PWAs, when working catches the requests coming from the frontend. The SW provides the Cache API, which is browser HTTP cache independent, and the 
IndexedDB database. To grant the offline work as well as a faster loading of the app, some parts of the app (the assests) are precached at the Cache API - there were saved the CSS and JS files - and some data is stored locally at the IndexedDB API. Instead of going directly to the server, the service worker retrieves the precached data in response to any request of the client $[1,6]$.

\section{Discussion}

The main setbacks of the chosen architecture are related to the PWA browser compatibility and the unavailability on app stores. For instance, Safari had SW compatibility not until this year. Although browsers are growing their support, there is still one feature that is only available on Google Chrome - the Background Sync API. However, this is a promissory feature allowing postponing actions until the user has internet connection. For that reason, the proposed app does not take advantage of it yet. Pregnant women can only edit data when connected.

Otherwise, all the buttons that lead to it are disabled and the user is informed that there is no internet connection. Secondly, the fact that PWAs aren't available on app stores might reduce their visibility since some users might use app stores as their primary search.

On the other hand, the scalability and the well functioning of the app are guaranteed by the technologies used to develop the frontend and backend as well as by the database chosen. Moreover, the UX provided by the chosen stack is enhanced by being a PWA instead of a classic mobile web app. By storing the assets on the Cache API and the database data on the IndexedDB the loading time will be lower after the first visit as well as it will be available even when offline. Besides, if the app is added to the mobile devices screen, it will behave like an ordinary mobile app.

\section{Conclusion}

After assessing the pros and cons of each type of the mobile apps and the advantages of the PWAs, it was proposed an EMR app. The proposed system has a set of tools that might be grouped on maternity record and appointments, health education, pregnancy-related information and monitoring tools. It was implemented as a PWA and the technologies used on it MongoDB, Express.js, React and Node.js - are ideal to develop a scalable and complex app. Besides, by implementing a PWA it does not take extra time and effort to have an app available to all the mobile platforms and computers. A uniform cross-platform experience, even without connectivity, is delivered by the proposed EMR.

In the future, a usability test must be executed to assay the value of the app for its users and where it can be improved. It might also be interesting to connect the proposed system to a hospital or maternity centre or even to evaluate how it is used and the impact it has on pregnant women.

\section{References}

[1] Introduction to service worker. https://developers.google.com/web/ilt/pwa/ introduction-to-service-worker, last viewed August 2018.

[2] React - a javascript library for building user interfaces. https://reactjs.org/, last viewed June 2018. 
[3] Mariam Bachiri, Ali Idri, José Luis Fernández-Alemán, and Ambrosio Toval. Mobile personal health records for pregnancy monitoring functionalities: Analysis and potential. Computer methods and programs in biomedicine, 134:121-135, 2016.

[4] Chung-Wei Chang, Tien-Yan Ma, Mei-San Choi, Yu-Yun Hsu, Yi-Jing Tsai, and Ting-Wei Hou. Electronic personal maternity records: both web and smartphone services. Computer methods and programs in biomedicine, 121(1):49-58, 2015.

[5] Alejandro Corbellini, Cristian Mateos, Alejandro Zunino, Daniela Godoy, and Silvia Schiaffino. Persisting big-data: The nosql landscape. Information Systems, 63:1-23, 2017.

[6] Google Developers. Offline quickstart. https://developers.google.com/web/ilt/pwa/ offline-quickstart, last viewed August 2018.

[7] Júlio Duarte, Carlos Filipe Portela, António Abelha, José Machado, and Manuel Filipe Santos. Electronic health record in dermatology service. In International Conference on ENTERprise Information Systems, pages 156-164. Springer, 2011.

[8] Wafaa S El-Kassas, Bassem A Abdullah, Ahmed H Yousef, and Ayman M Wahba. Taxonomy of cross-platform mobile applications development approaches. Ain Shams Engineering Journal, 8(2):163-190, 2017.

[9] Gunther Eysenbach and Thomas L Diepgen. The role of e-health and consumer health informatics for evidence-based patient choice in the 21st century. Clinics in dermatology, 19(1):11-17, 2001.

[10] Francisca Fonseca, Hugo Peixoto, Filipe Miranda, José Machado, and António Abelha. Step towards prediction of perineal tear. Procedia Computer Science, 113:565-570, 2017.

[11] Irini Genitsaridi, Haridimos Kondylakis, Lefteris Koumakis, Kostas Marias, and Manolis Tsiknakis. Evaluation of personal health record systems through the lenses of ec research projects. Computers in biology and medicine, 59:175-185, 2015.

[12] Misha Kay, Jonathan Santos, and Marina Takane. mhealth: New horizons for health through mobile technologies. World Health Organization, 64(7):66-71, 2011.

[13] Patrícia Loreto, Francisca Fonseca, Ana Morais, Hugo Peixoto, António Abelha, and José Machado. Improving maternity care with business intelligence. In Future Internet of Things and Cloud Workshops (FiCloudW), 2017 5th International Conference on, pages 170-177. IEEE, 2017.

[14] Ivano Malavolta. Beyond native apps: web technologies to the rescue!(keynote). In Proceedings of the 1st International Workshop on Mobile Development, pages 1-2. ACM, 2016.

[15] Marie Gunnel Oscarsson, Erica Medin, Ida Holmström, and Lena Lendahls. Using the internet as source of information during pregnancy-a descriptive cross-sectional study among fathers-to-be in sweden. Midwifery, 62:146-150, 2018.

[16] Linda G Park, Alexis Beatty, Zoey Stafford, and Mary A Whooley. Mobile phone interventions for the secondary prevention of cardiovascular disease. Progress in cardiovascular diseases, 58(6):639$650,2016$.

[17] Hugo Peixoto, Manuel Santos, António Abelha, and José Machado. Intelligence in interoperability with aida. In International Symposium on Methodologies for Intelligent Systems, pages 264-273. Springer, 2012.

[18] Joachim Perchat, Mikael Desertot, and Sylvain Lecomte. Component based framework to create mobile cross-platform applications. Procedia Computer Science, 19:1004-1011, 2013.

[19] Ana Pereira, Fernando Marins, Bruno Rodrigues, Filipe Portela, Manuel Filipe Santos, José Machado, Fernando Rua, Álvaro Silva, and António Abelha. Improving quality of medical service with mobile health software. Procedia Computer Science, 63:292-299, 2015.

[20] Vasan Subramanian. Pro MERN stack: full stack web app development with Mongo, Express, React, and Node. Springer, 2017.

[21] Robyn Whittaker, Hayden McRobbie, Chris Bullen, Ron Borland, Anthony Rodgers, and Yulong Gu. Mobile phone-based interventions for smoking cessation. Cochrane Database Syst Rev, 2012. 